\title{
Value of volume weighted mean nuclear volume in grading and prognosis of renal cell carcinoma
}

\author{
E Artacho-Pérula, R Roldán-Villalobos, J F Martínez-Cuevas
}

\begin{abstract}
Aims-To perform stereological quantitation of volume weighted mean nuclear volume in renal cell carcinomas; and to correlate the data obtained with recognised clinical and pathological variables and determine their prognostic value.

Methods-The point-sampled intercepts method was used to estimate mean nuclear volume in 63 cases of clear cell renal carcinoma diagnosed between 1980 and 1988. New paraffin wax embedded histological sections were analysed after systematic sampling and the test systems superimposed on a projected microscopic image to measure nuclear intercept lengths. After mathematical estimation of mean nuclear volume, statistical analyses of the data in relation to clinical and pathological variables as well as the prognostic impact were investigated.
\end{abstract}

Results-The mean nuclear volume was significantly associated with tumour dedifferentiation. However, mean nuclear volume showed no statistical differences with sex, age, and clinical stage. The prognostic value of mean nuclear volume, nuclear grading, and clinical stage in renal cell carcinomas was high: mean nuclear volume greater than $140 \mu \mathrm{m}^{3}$ was associated with short term survival.

Conclusions-Measurement of mean nuclear volume was useful as a guide to objective grading of renal cell carcinomas, though there was an overlap between tumour grades. Based on the limited number of cases analysed, the mean nuclear volume is proposed as an additional prognostic indicator.

(F Clin Pathol 1994;47:324-328)

Clear cell renal carcinoma is a carcinoma of renal tubular cells. It comprises a high percentage of diagnosed renal carcinomas. Classification of these tumours is currently based on the evaluation of the clinicopathological characteristics which are often very difficult to reproduce. Thus the degree of tumour cell anaplasia is used in grading histological specimens, ${ }^{1}$ with grades 1 and 4 having the least and most severe degree of anaplasia, respectively; grades 2 and 3 are intermediate. Patients are frequently diagnosed as having these latter grades of tumoural dedifferentiation.
Three features influence prognosis ${ }^{1}$ : tumour volume, extension or clinical stage; and histomorphological characteristics. Renal cell carcinoma has a poor prognosis because it is often diagnosed only after metastasis. ${ }^{2}$ The histological diagnosis is based on the clinician's experience and the subjective opinion of the pathologist. These can result in low diagnostic reproducibility. In recent years quantitative techniques have been used to avoid subjective diagnosis.

Quantitative methods have three features ${ }^{3}$ : objectivity; reproducibility; and a capacity to detect alterations that facilitate improved patient diagnosis. Morphometry also has an important role in clarifying "borderline" cases. ${ }^{3}$ The tumour cell nucleus is the most widely used quantitative variable: it supplies biologically relevant data which can be correlated with histopathological grade and patient prognosis. ${ }^{45}$ Concerning the quantitation of nuclear size, Gundersen and Jensen ${ }^{6}$ recently reported a new stereological method called the point-sampled intercepts method for estimation of the volume weighted mean nuclear volume from ordinary histological sections.

\section{Methods}

Seventy four cases of renal cell carcinoma diagnosed between 1980 and 1988 were retrieved from the files of the pathology service of the Reina Sofia Hospital, Córdoba, Spain. The paraffin wax embedded blocks were retrieved and new histological sections prepared ( $3 \mu \mathrm{m}$ thick) and stained with haematoxylin and eosin. It was assumed that the routine processing and sectioning of the specimens were done randomly and thus isotropic uniform random sections were obtained. ${ }^{7}$

Diagnosis and grading were confirmed by two pathologists according to the criteria of Furhman et al. ${ }^{1}$ Poorly visible samples and those in which there was no diagnostic agreement between pathologists were eliminated from the study, and so 63 cases of clear cell renal carcinoma (grade $1, \mathrm{n}=1$; grade 2 , $\mathrm{n}=44 ;$ grade $3, \mathrm{n}=16$; and grade $4, \mathrm{n}=2$ ) were included in this study. The clinical features of all patients are summarised in table 1 .

\section{STEREOLOGICAL METHODS}

A Leitz ASM semiautomatic image analysis system in association with a Leitz-Dialux 20 microscope were used to perform the 
Table 1 Summary of clinical data

\begin{tabular}{|c|c|c|c|c|c|c|c|c|c|c|c|c|c|c|}
\hline \multirow{2}{*}{$\begin{array}{l}\text { Nuclear } \\
\text { grade }\end{array}$} & \multirow{2}{*}{$\begin{array}{l}\text { No of } \\
\text { patients }\end{array}$} & \multicolumn{2}{|l|}{ Sex } & \multicolumn{6}{|c|}{ Age (years) } & \multicolumn{5}{|c|}{ Clinical stage } \\
\hline & & Women & Men & $31-40$ & $41-50$ & $51-60$ & $61-70$ & $71-80$ & $81-90$ & $I$ & II & III & IV & ? \\
\hline $\begin{array}{l}1 \\
2 \\
3 \\
4\end{array}$ & $\begin{array}{r}1 \\
44 \\
16 \\
2\end{array}$ & $\begin{array}{r}1 \\
28 \\
8 \\
2\end{array}$ & $\begin{array}{r}16 \\
8\end{array}$ & $\begin{array}{l}1 \\
2\end{array}$ & $\begin{array}{l}7 \\
1\end{array}$ & $\begin{array}{r}1 \\
12 \\
4\end{array}$ & $\begin{array}{r}14 \\
8\end{array}$ & $\begin{array}{l}9 \\
2 \\
1\end{array}$ & 1 & $\begin{array}{r}1 \\
24 \\
6\end{array}$ & $\begin{array}{l}5 \\
1 \\
1\end{array}$ & $\begin{array}{l}9 \\
2 \\
1\end{array}$ & $\begin{array}{l}4 \\
6\end{array}$ & $\begin{array}{l}2 \\
1\end{array}$ \\
\hline Total & 63 & 39 & 24 & 3 & 8 & 17 & 22 & 12 & 1 & 31 & 7 & 12 & 10 & 3 \\
\hline
\end{tabular}

quantitative analysis. A final magnification of $\times 1250$ was used after system calibration. Individual nuclear size was obtained using the point-sampled intercepts method described by Gundersen and Jensen. ${ }^{6}$ The volume weighted mean nuclear volume was estimated following systematic sampling within the section (fig 1). A rectangular counting frame and a test system composed of parallel lines with

Figure 1 Selected fields of vision (1) following systematic sampling of the slice. Continuous line represents the section of renal tumour.
Figure 2 (A) Test system composed of points associated with parallel lines on counting frame including the "forbidden line" left hand and bottom edges) to show the pointsampled intercepts method. Each nuclear intercept length was measured (thick segment) to estimate mean nuclear volume. (B) Field of vision from a grade 2 clear cell renal carcinoma with the test system

superimposed

(haematoxylin and eosin).

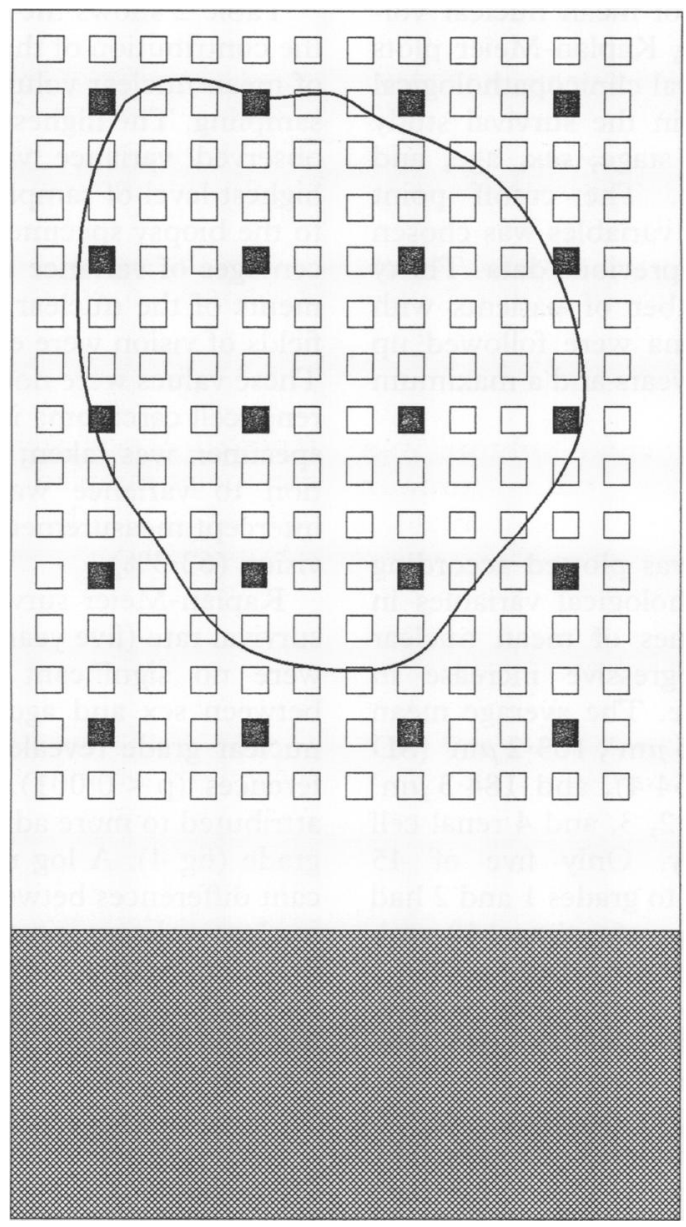

equidistant intercept points was superimposed on each systematically selected field of vision (fig 2). Any arbitrary direction of the test lines was used because nuclei have no preferred direction of orientation in isotropic uniform random sections. If a point hits a nuclear profile the intercept through the sampling point is measured from nuclear boundary to nuclear boundary in the direction of the test line. When a nucleus was hit two or more times, the intercept length was also measured two or more times; thus the intercept length must be measured each time the test points hit the nucleus. However, the test system was constructed for nuclei generally only hit by one test point. Each intercept length $\left(l_{0}\right)$ is then raised to the power of three. The mean of the individually cubed intercept lengths $\bar{l}_{0}^{3}$ multiplied by $\pi / 3$ is an unbiased estimate of the mean nuclear volume. For each biopsy specimen, a mean number of 100 (range 62 to 192) nuclear intercept lengths were measured in an average of seven fields of vision (range four to eight). It took about 15 minutes to measure each biopsy specimen.

\section{STATISTICAL ANALYSIS}

Estimates of mean nuclear volume were calculated with an IBM-PC compatible microcomputer using a program designed by us in combination with Statistical Analysis System (SAS). The same program provides information of the contribution from different levels of sampling to the total observed variance of mean nuclear volume by the method of nested analysis of variance. ${ }^{8}$ Comparisons of averaged estimations of mean nuclear volume were carried out with respect to all clinicopathological variables. If a group had a small number of samples they were included in the nearest group. Group mean values of mean nuclear volume were compared using Student's $t$ test. One-way analysis of variance
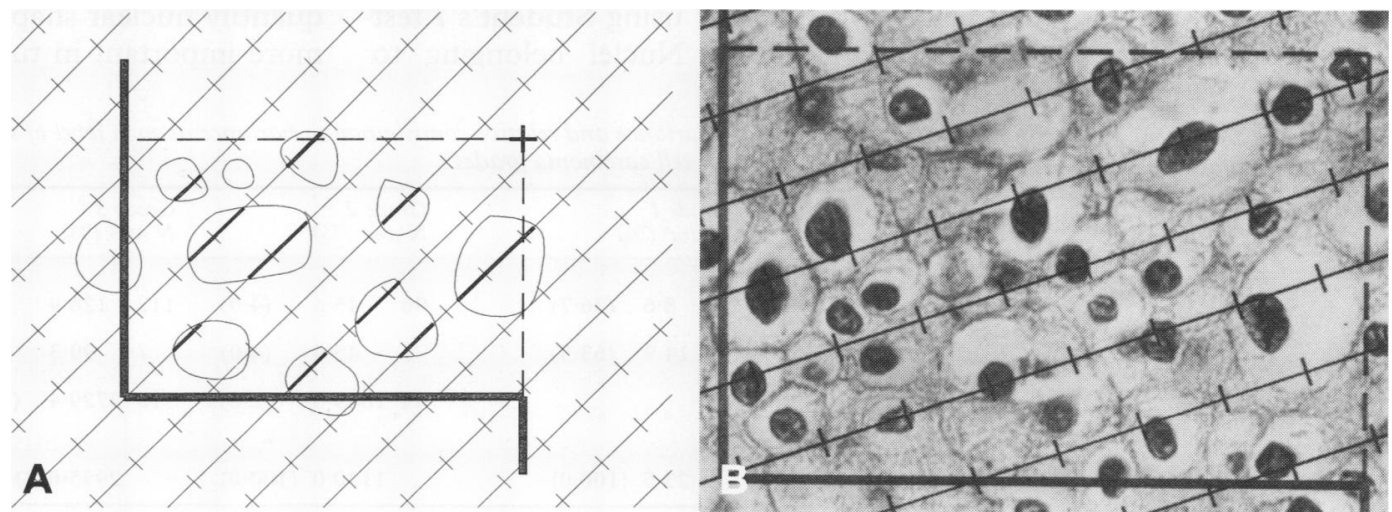
Figure 3 Plot of the mean nuclear volume estimates against clinicopathological variables of 63 biopsy specimens with renal cell carcinoma. Horizontal and vertical bars indicate mean $(S D)$.

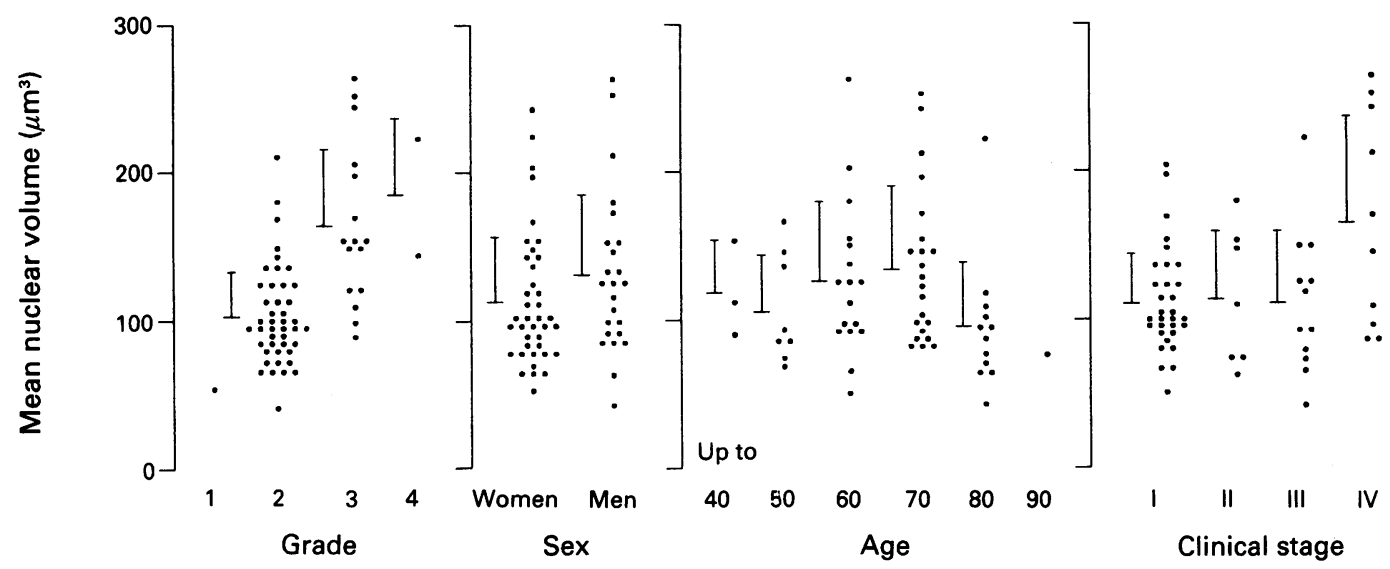

in association with Scheffe's procedure was used for the comparison between the means of more than two groups. ${ }^{9}$ In case of a lack of homogeneity of variances a non-parametric Kruskal-Wallis test was used.

The prognosis study of mean nuclear volume was analysed using Kaplan-Meier plots and log rank tests. Several clinicopathological features were included in the survival study (nuclear grade, clinical stage, sex, age, and mean nuclear volume). The cutoff point selection of quantitative variables was chosen after an examination of previous data. Thirty seven of the total number of patients with clear cell renal carcinoma were followed up for a minimum of three years and a maximum of 11 .

\section{Results}

Mean nuclear volume was plotted according to individual clinicopathological variables in fig 3. The average values of mean nuclear volume showed a progressive increase in relation to nuclear grade. The average mean nuclear volume was $53.5 \mu \mathrm{m}^{3}, 103.2 \mu \mathrm{m}^{3}$ (SD 33.8), $164.0 \mu \mathrm{m}^{3}$ (SD 54.4), and $184.3 \mu \mathrm{m}^{3}$ (SD $54 \cdot 8$ ) for grades $1,2,3$, and 4 renal cell carcinomas, respectively. Only five of 45 $(11 \cdot 1 \%)$ cases belonging to grades 1 and 2 had a mean nuclear volume greater than $140 \mu \mathrm{m}^{3}$; $27 \cdot 8 \%$ of renal cell carcinoma cases grades 3 and 4 had a mean nuclear volume smaller than $140 \mu \mathrm{m}^{3}$. The coefficients of error estimation of mean nuclear volume were $4.9 \%$, $8.3 \%$, and $21.0 \%$ for grades 2,3 , and 4 renal cell carcinomas, respectively. Significant differences were shown between grades 1 and 2 with respect to grades 3 and 4 renal cell carcinomas using Student's $t$ test $(t=4.75, \mathrm{p}<0.001)$. Nuclei belonging to grades 3 and 4 renal cell carcinomas were greater than grades 1 and 2 by a factor of 1.63. In contrast, estimates of mean nuclear volume, grouped according to clinical stage, sex, and age were not significantly different.

Table 2 shows the results of the analysis of the contribution of the total observed variance of mean nuclear volume for different levels of sampling. The highest contributor to the total observed variance was associated with the highest level of sampling which corresponded to the biopsy specimens. In contrast, the percentages of variance observed from measurements of the nuclear intercepts and different fields of vision were equal to or less than $8 \%$. These values were notably different in grade 1 renal cell carcinoma in which only one biopsy specimen was taken; thus the total contribution to variance was provided by nuclear intercept measurements $(36 \cdot 7 \%)$ and fields of vision $(63 \cdot 3 \%)$.

Kaplan-Meier survival analysis indicated a survival rate (five years) of $68 \%$ (fig 4 ). There were no significant differences in survival between sex and age, but clinical stage and nuclear grade revealed highly significant differences $(p<0.001)$. A poor prognosis was attributed to more advanced clinical stage and grade (fig 4). A log rank test showed significant differences between patients with a mean nuclear volume greater than $140 \mu \mathrm{m}^{3}$ and a mean nuclear volume equal to or less than $140 \mu \mathrm{m}^{3}$. The mortality rate was higher in patients with a nuclear volume greater than $140 \mu \mathrm{m}^{3}$ (fig 4).

\section{Discussion}

The morphometric variables that define and quantify nuclear shape and size are becoming more important in tumour pathology, in that

Table 2 Absolute observed variance and relative contribution to variance at each level of sampling for volume weighted mean nuclear volume in renal cell carcinoma grades

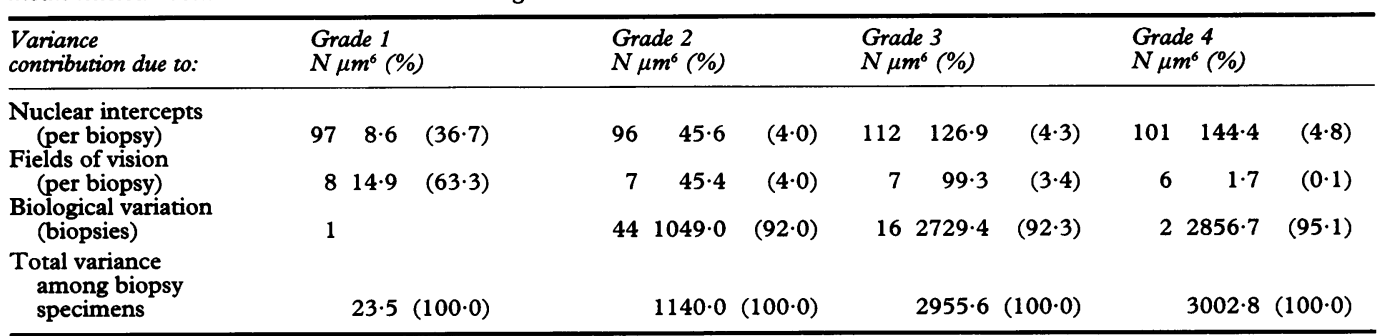


Figure 4 Survival of 37 patients with renal cell carcinomas according to estimates of mean nuclear volume and clinicopathological variables.
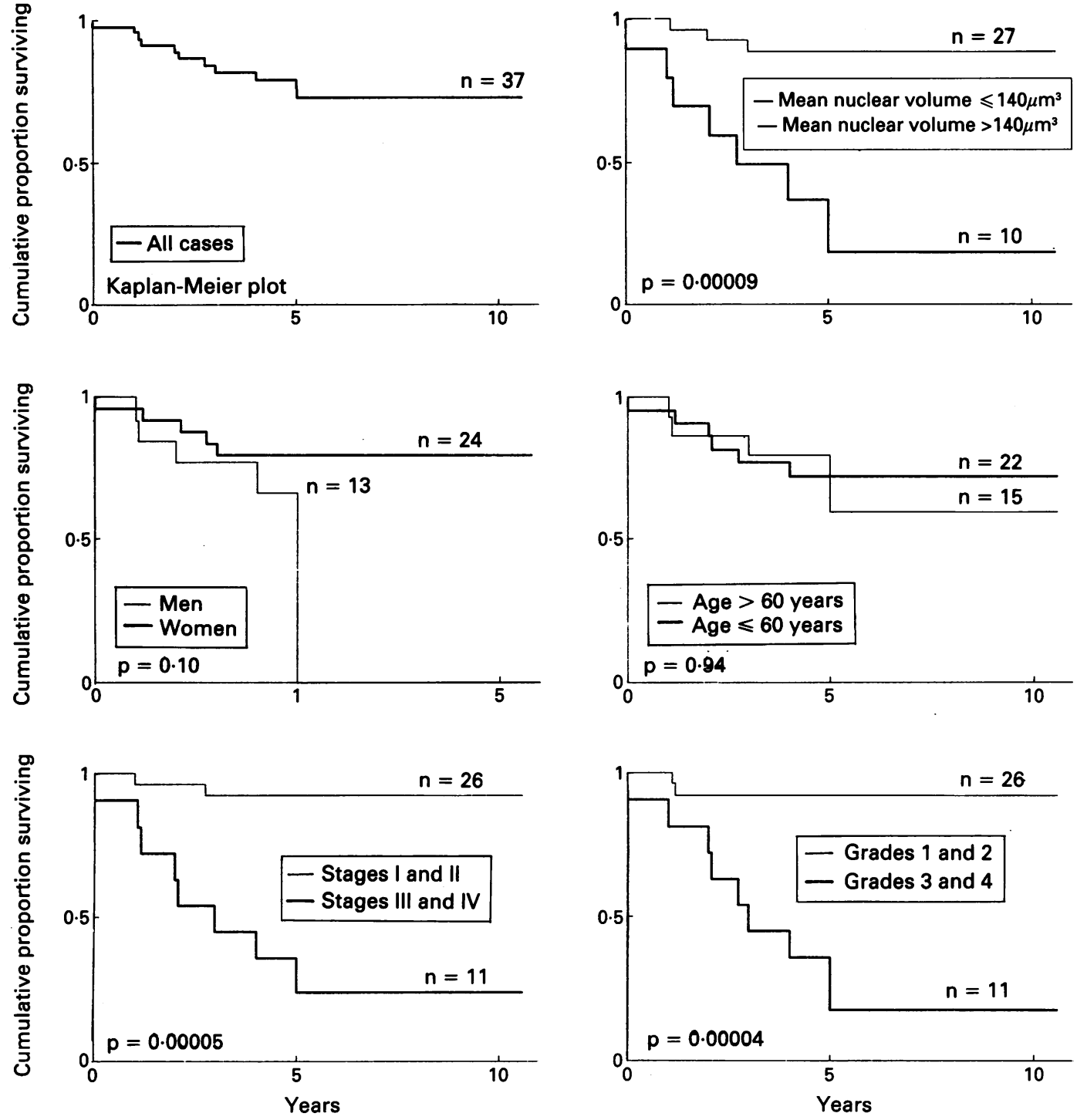

nuclear morphometry is an important predictive factor of neoplastic potential. ${ }^{10} 11$ The most habitually used variable is the nuclear area, but new methods have appeared that permit measurement of the nuclear volume, and because it is a three-dimensional structure, it seems more appropriate to quantify its volume than its area.

The estimation of the mean nuclear volume by the point-sampled intercepts method, ${ }^{6}$ apart from being an unbiased and realistic variable of three-dimensional nuclear size, is associated, statistically, with the qualitative grading of renal carcinoma samples, in which nuclear volume increases with tumour dedifferentiation. The easy and fast estimation of mean nuclear volume, the simple requirement of the unambiguous identification of the nuclei, and its feature of being a shape independent size descriptor of the nuclei, should be very useful to pathologists. The advantages of the mean nuclear volume over two-dimensional variables (area, perimeter, shape factors) are evident. The efficiency of the mean nuclear volume estimation in different pathological entities has been widely demonstrated. ${ }^{12-14}$ Furthermore, these estimates are highly sensitive for detecting slight increases in nuclear size because the method is based

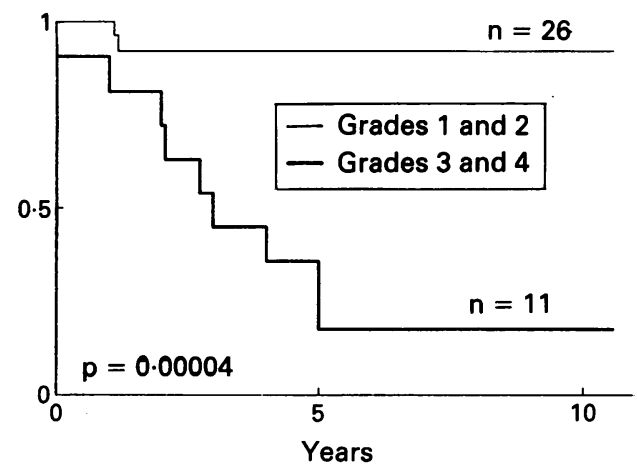

on point-sampling and thus there is greater probability of sampling larger than smaller nuclei. Another feature of the mean nuclear volume estimates is to provide information on nuclear size variability and, consequently, nuclear size pleomorphism.

The analysis of the contribution to total observed variation of mean nuclear volume and time required shows the high efficiency and precision of the procedure following simple sampling. Biological variation between individual renal cell carcinoma grades contributed to more than $90 \%$ of the total variance. The numbers of measured intercept lengths have little influence on the total variance, and heterogeneous tumours were not seen as field variation was not a significant factor. Thus there is no need to carry out more measurements and increase fields of vision with a corresponding increase in time.

Similar to others, our study found a $68 \%$ survival rate in patients with renal cell carcinoma. ${ }^{4515}$ Sex and age were independent factors in patient outcome. Clinical stage and histological grade of renal cell carcinoma samples provide prognostic information. In both, advanced stage and grade were associated with short term survival. Different quantitative variables were correlated with patient 
survival. Thus Tosi et al ${ }^{14}$ found that assessment of the nuclear area was a good prognostic factor in stage I renal cell carcinomas, while nuclear shape was a less powerful discriminator in patient survival. The threedimensional mean nuclear volume provided significant prognostic information; using a cutoff point of $140 \mu \mathrm{m}^{3}$, mean nuclear volume estimates permit the establishment of different patient prognosis in renal cell carcinomas. The prognosis impact of mean nuclear volume has been shown in several entities (papillary carcinomas of the ampulla of Vater, ${ }^{12}$ bladder cancer, ${ }^{13}$ cutaneous malignant melanomas, ${ }^{14}$ squamous cell carcinomas of the uterine cervix ${ }^{17}$ ), while Bryne et al ${ }^{18}$ found that the prognosis of mean nuclear volume estimates in oral carcinomas was of no value.

We are grateful to $R$ Vaamonde-Lemos, and $F$ López-Rubio, for histopathological evaluation of samples. The excellent technical and photographic assistance of $\mathbf{P}$ SepulvedaMadueño and E Tarradas-Merino is also greatly appreciated.

1 Furhman SA, Lasky LC, Limas C. Prognostic significance of morphologic parameters in renal cell carcinoma. $A m^{\mathcal{F}}$ Surg Pathol 1982;6:655-63.

2 Eker R, Nesland JM, Andersen A, Johannesnssen JV. Prognostic factors in renal cell carcinoma. Histol Histopathol 1986;1:255-70.

3 Baak JPA, Oort J. In: Baak JPA, Oort J, eds. A manual of morphometry in diagnostic pathology. Berlin: SpringerVerlag, 1983:1-46.

4 Bibbo M, Galera-Davidson H, Dytch HE. Karyometry and histometry of renal cell carcinoma. Anal Quant Cytol Histol 1987;9:182-7.
5 Gonzalez-Campora R, Gonzalez-de-Chaves FJ, MoraMartin J. Nuclear planimetry in renal-cell tumors. Anal Quant Cytol Histol 1991;13:54-60.

6 Gundersen HJG, Jensen EB. Stereological estimation of the volume-weighted mean volume of arbitrary particles observed on random section. $f$ Microsc 1985;131 291-310.

7 Gundersen HJG, Bendtsen TF, Korbo L, et al. Some new, simple and efficient stereological methods and their use in pathological research and diagnosis. APMIS 1988;96:379-94.

8 Gundersen HJG, Østerby R. Optimizing sampling efficiency of stereological studies in biology: or 'Do more ciency of stereological studies in biology
less well!!'. ₹ Microsc 1981;121:65-73.

9 Scheffe H. The analysis of variance. New York: John Wiley, 1959.

10 Ambros RA, Trost RC, Campbell AY, Lambert WC. Prognostic value of morphometry in papillary thyroid carcinoma. Hum Pathol 1989;20:215-8.

11 Diamond DA, Berry SJ, Jewett HJ, Eggleston JC, Coffey D. A new method to assess metastatic potential of human prostate cancer: relative nuclear roundness. $f$ Urol 1982;128:729-34.

12 Artacho-Pérula E, Roldán-Villalobos R, López-Rubio F, Vaamonde-Lemos R. Stereological estimates of nuclear volume in carcinoma of the ampulla of Vater. volume in carcinoma of
Histopathology 1992;21:241-8.

13 Nielsen $K$, Colstrup H, Nilsson T, Gundersen HJG Stereological estimates of nuclear volume correlated with histopathological grading and prognosis of bladder tumor. Virchows Arch (Cell Pathol) 1986;52:41-54.

14 Sørensen FB. Objective histopathological grading of cutaneous malignant melanomas by stereological estimation of nuclear volume. Prediction of survival and diseasefree period. Cancer 1989;63:1784-98.

15 Garofalo F, Messina S, Santis P, Nanni G. Características clínicas y patológicas del carcinoma de riñón: análisis computerizado. Arch Esp Urol 1991;44:691-5.

16 Tosi P, Luzi P, Baak JPA, et al. Nuclear morphometry as an important prognostic factor in stage I renal cell carcian important prognostic factor

17 Sørensen FB, Bichel P, Jakobsen A. DNA-level and stereological estimates of nuclear volume in squamous cell carcinoma of the uterine cervix: a comparative study with analysis of prognostic impact. Cancer 1992;69:187-99.

18 Bryne M, Nielsen K, Koppang HS, Dabelsteen E. Grading of nuclear pleomorphism in oral carcinomas. Higher prognostic value than stereological assessment of nuclear volume. APMIS 1991;99:333-9. 